\title{
Supporting Interactive Graph Analysis Using Adjustable Magic Lenses
}

Ulrike Kister

Interactive Media Lab

Technische Universität Dresden

Dresden, Germany

ukister@acm.org

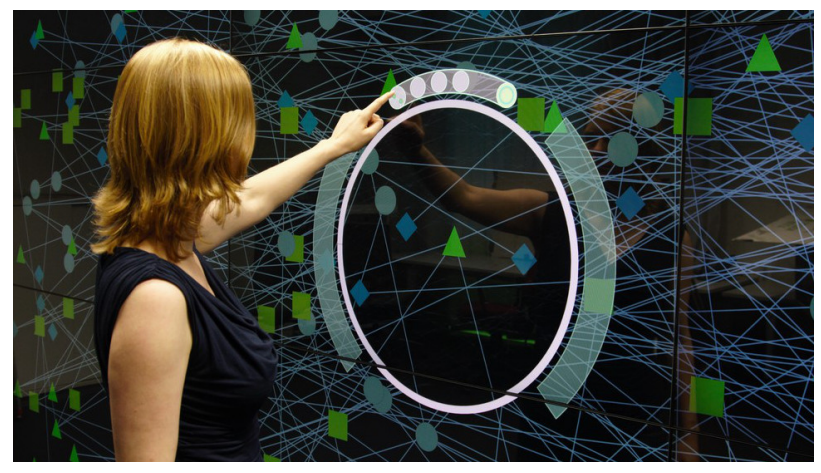

Figure 1: Magic lenses can be used to explore and edit graphs. Their adjustment with natural user interfaces is part of this work.

Permission to make digital or hard copies of part or all of this work for personal or classroom use is granted without fee provided that copies are not made or distributed for profit or commercial advantage and that copies bear this notice and the full citation on the first page. Copyrights for third-party components of this work must be honored. For all other uses, contact the owner/author(s).

Copyright is held by the author/owner(s).

ITS 2015, November 15-18, 2015, Funchal/Madeira, Portugal.

ACM 978-1-4503-3899-8/15/11.

http://dx.doi.org/10.1145/2817721.2820989

\begin{abstract}
Magic lenses are important tools for visualization and can be used to explore and edit graphs. Here, large displays can be beneficial for both their visualization space and possibility for multi-user analysis. With this work, we contribute the enhancement of graph lens functionality by allowing the user to switch lens functions, adjust function parameters, and combine lenses. Further, we investigate the application of various interaction modalities for magic lenses, such as multi-touch, tangibles, and body-centric interaction, to support interactive graph exploration and manipulation.
\end{abstract}

\section{Author Keywords}

Magic lenses; information visualization; multi-touch; embodied interaction; graphs; territoriality.

\section{ACM Classification Keywords}

H.5.2. [Information Interfaces and Presentation (e.g. $\mathrm{HCl}$ )]: User Interfaces

\section{Introduction}

Graph data is essential in various domains, e.g., biology, computer science, or communication technology. There it is important to explore and edit graph data, their nodes, edges and individual attributes. Magic lenses are user interface tools that apply a function to data in a region of interest changing the view [2]. They have been used for diverse 
tasks and data types in visualization [17] and can also be applied to graph representations (e.g., [16]). Most of these implementations have been designed for the desktop environment. However, novel large display environments provide tremendous benefits. The display space alone allows for more visualization space, so that larger sets of nodes or alternative representation of the data can be presented at once. Furthermore, these setups allow multiple users to explore and manipulate graphs collaboratively or in-parallel. In these scenarios, natural user interfaces are required as they support direct interaction while moving in front of the display. Here, both interaction on and in front of the display can be used to manipulate the visualization.

In my thesis, I investigate interactive exploration and manipulation of graphs and the use of magic lenses for this purpose (Figure 1). Within this topic, I plan to address various interaction modalities, e.g., multi-touch, tangibles, body-centric interaction, mobile device gestures, and gaze. I specifically focus on the individual properties, functions and function parameters of magic lenses and how they can be fluently adjusted by the user with appropriate interaction modalities and gestures.

The following section presents a selection of existing research and background on magic lenses. Afterwards, our prior work and the on-going research for this thesis are described. Finally, the future research plan and next steps are presented.

\section{Background and Existing Research}

This section aims at giving a short summary of the basic principles and properties of magic lenses and existing interaction approaches. A thorough overview is given in our state-of-the-art report [17] of which the following can only address few selected points.
For user interfaces, magic lenses were first defined by Bier et al. [2] and change the view within a region of interest. Their properties position, size and shape, and orientation make up their main geometry [17]. The lens' shape is fixed or changes dynamically. Fixed shape are the most commonly used and are often elliptical $[11,16]$. Dynamic shapes are either defined by the content [12] or by the user, e.g., by sketching the region of interest in advance [3].

The most important aspect of the lens is its function. There have been more than 40 lens functions presented in literature for various tasks and data types [17], also for graph data (e.g., $[13,16])$. However, they are presented as lenses with a single function. An exception is the Composite Lens [16] where a fixed combination of three functions is presented as a new lens. Additionally, to adjust the individual lens function (if at all possible) additional GUI dialogs are used. In the simple case of magnification the zoom factor is such a parameter to adjust. For more advanced lens functions, parameters such as degree of adjacency for Bring Neighbors Lenses [16] or filter parameters may need to be set. These parameters can be of various type (continuous, discrete, multiple-selections) and their adjustment can have an essential influence on the resulting outcome of the lens.

There has been research using magic lenses on interactive surfaces. As a first touch-enabled graph lens, the Push-

Lens [13] declutters the region of interest by pushing edges from the lens. Butscher et al. [5] use magnification lenses for collaborative image exploration. In a demo, Lander et al. [10] present a map exploration with magnification lenses. However, while all these lenses can be dragged (and often resized) to adjust their selected region of interest, they cannot be adjusted further in selecting other appropriate lens function(s) or even setting individual parameters of the function. 


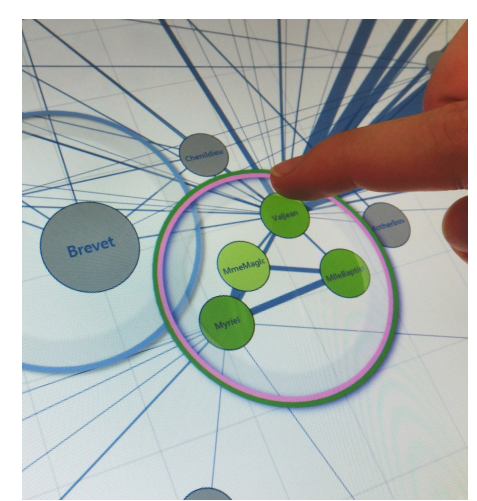

Figure 2: Multi-touch enabled magic lenses with various lens functions for graph exploration [8].

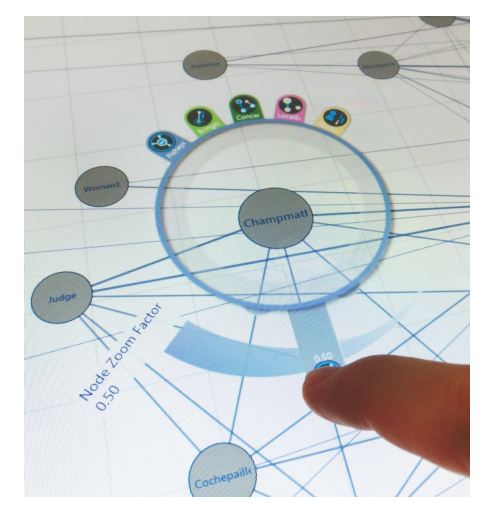

Figure 3: Parametrizing and adjusting multi-touch lenses in place [8].
Making magic lenses graspable, Kim and Elmqvist [7] use thin transparencies on tabletops and this way allow stacking of lenses. Even more so, Spindler et al. [15] use spatial interaction with magic lenses for information visualization. Among other applications, they use the distance to a tabletop surface to expand hierarchical nodes in a graph. They present an overview of a possible interaction vocabulary with tangible magic lenses above tabletops, which is likely to be adaptable to vertical displays. In front of a vertical display, Lehmann et al. [11] use head tracking to visualize a lens at the user's view point. However, again this lens is fixed in its shape and function and cannot be adjusted further by the user.

\section{Goals and Expected Contributions}

The main goal of this work will be to improve interaction with magic lenses using graph exploration and editing as the main application case. However, the developed concepts will also be generalized and made usable for other data exploration and editing tasks. In enhancing magic lenses, we aim at the following research goals:

(G1) Developing the magic lens as a tool with more than one lens function, that can be interchanged, parametrized and adjusted by the user.

(G2) Finding and combining appropriate interaction modalities to adjust the lens (in various degrees of granularity) within the user's workflow on and in front of large, interactive vertical displays.

(G3) Extending the magic lens from a view onto the data to a personal territory with individual tools supporting collaborative work.

\section{Completed and On-going Work}

Our approach to address this research started with an analysis of magic lenses, existing lens functions and especially the various interaction modalities that have been applied to implement interactive magic lenses in existing works [17]. We identified open research questions and found that especially the combination and parametrization of lenses has been neglected in research so far. As a consequence, we investigate various modalities, their appropriateness for magic lenses, and the possibilities to adjust and adapt the lens, its function(s), and parameters.

Investigating Function Parameter Adjustment using Multi-touch To address our goals G1 and G2, we designed touch-enabled magic lenses that differ from previous work in that i) they allow flexible switches of lens functions within one lens, ii) lenses can be combined to include multiple lens functions, and iii) they allow explicit adjustment of the lens function parameters [8].

The lenses can be positioned and resized using multi-touch gestures (Figure 2), similar to previous work. However, we designed various novel ways of manipulating lens function and parameters. For externalizing the various possible graph lens functions that can be triggered within the lens, we have menus at the top along the border. Tapping or sliding these menu icons switches the lens function. Further, for each graph lens function, we identified its parameters. These can be adjusted in-place at the lower border of the lens (Figure 3). We implemented discrete and continuous widgets to adjust these parameters in place along the border. In the design process, we enforced use of spatial memory as menu items are always placed in the same location, and relative adjustment of values, as the slider adapts its position to the current value. For expert users, we also developed continuous gestures that allow fluent adjustment of both lens function and one primary parameter without lifting the fingers [8]. Further, we allow flexible combination of multiple lenses as dragging the lenses on top of each other 


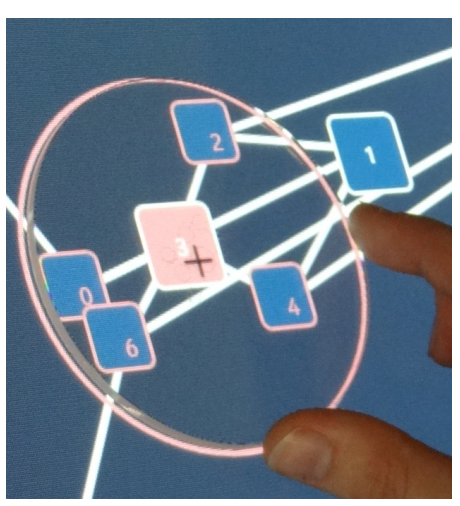

Figure 4: Transparent tangibles support use as magic lenses $[4,6]$

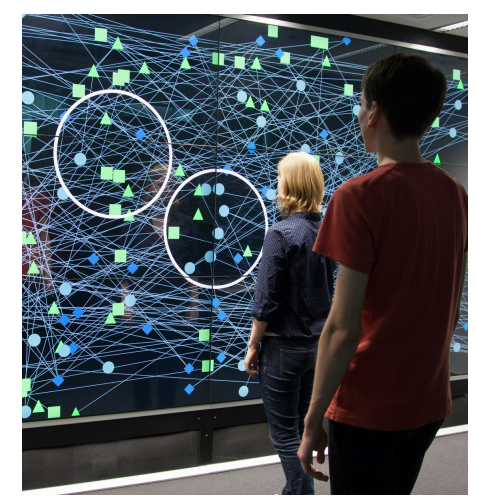

Figure 5: Coupling the lens' position with the user's body can expand the lens' capabilities to a personal territory and embodied tool [9]. unites them to form one lens with multiple functions. We conducted a small pilot study to confirm usability. However, further work is required to understand long-term use of the tool by domain experts.

\section{Addressing the Benefits of Graspable Magic Lenses}

To improve use of multiple lenses by a single user, we examined the possibilities of making lenses tangible and thereby easier to grasp than touch-enabled virtual lenses. As by definition the lens' content is the main focus of the user, we looked at transparent tangibles as possible magic lenses [6] addressing our goal G2 (Figure 4). To first understand the properties of transparent tangibles, we reviewed the related work and defined a design space focusing on various properties, such as form factors, materials, role and function, and visualization space [4]. We identified potential benefits, e.g., precise positioning, that can be relevant for the application as magic lenses and investigated various application cases for transparent tangibles.

Using an interaction vocabulary similar to Spindler et al. [15], tangible lenses can be translated, rotated, flipped and interacted upon, as well as stacked. For transparent tangibles, we found that depending on tracking technology touch on the lens is possible. This is essential for magic lenses for graph exploration and editing as the data not the lens should be explored. In contrast to thin transparencies [7], thicker plates are better graspable. However, stacking of these lenses for combination is quite limited (in our setup: three tangibles) and requires additional tracking capabilities to be improved to still allow touch through the stack.

Thus far, we investigated magic lens functions, their combination and adjustment. However, we plan to further enhance the lens to be more than a tool for visualization but a collection of personal appliances and an all around analysis and editing tool.
Transforming Magic Lenses to Embodied, Personal Territories The focus of our current work is the embodiment of magic lenses on large display walls, addressing goals G2 and G3. In our BodyLens concept [9], the user's body is tracked and is applied as input for lens adjustment. The lens' position is coupled to the user's movement. Thereby, the lens is always placed in front of the user to ensure that its region of interest coincides with where the user is working. Thereby the lens becomes more than a tool, but a personal work territory. In a multi-user scenario, the lens visualizes the user's position and other user's are made aware of which nodes and edges are currently handled by the user (Figure 5). Further, we apply the principles of proxemic interactions [1] and use dimensions such as position, distance, and orientation to adjust lens parameters.

As the user's body is tracked using the Kinect One, this input can also be used to influence the shape of the lens. Different stages of mapping the user's body form to the lens shape are possible. While presenting a human-shadow shape [14] makes the personalization of the lens obvious, this does not seem convenient for graph exploration and manipulation. Furthermore, we found that shapes that constantly react to the user's movement, e.g., when being coupled with hand positions, will take focus away from the data to be explored. We tested various stages of embodiment for the lens shapes and found the reduction of movement by not mapping very active extremities convenient. We hypothesize that a bullet shape (see Figure 6) shadowing the user but being convex and leaving a large center for observation and manipulation of the data, is most suitable. However, this has still to be confirmed in a user study.

Looking at the proxemic dimensions, the lens can further be influenced by the user's distance to the display. This distance can be used for lens properties, such as shape 


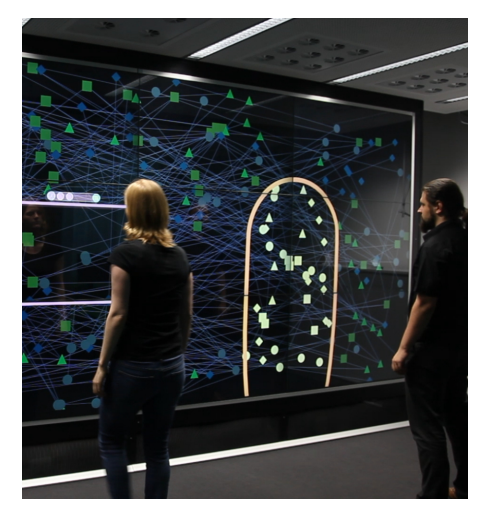

Figure 6: When the user's body is tracked, dynamic, user-dependent lens shapes and contents are possible [9].

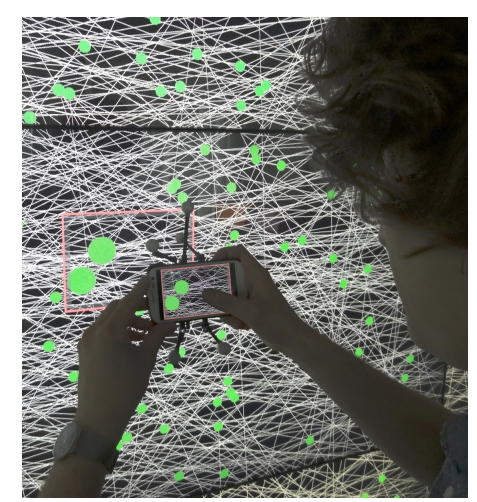

Figure 7: In the future, we want to develop mobile magic lenses that allow even more personalized views. or size, e.g., the lens gets larger as the user steps toward the display as he/she occludes and occupies the data to work on. Further, the distance can be used to change a lens function parameter, similar to the sliders presented for multi-touch, a user can continuously change a value. For magnification this means the content is zoomed more (or less) when the user steps toward the content. An interesting aspect in this context is the interaction granularity when changing values. Depending on the context the user might want to casually stroll changing values in coarse grained steps to explore the data set. In other situations, precision might be key and transitions between both are necessary. These differences have yet to be investigated and the appropriate interaction modalities and gestures selected.

\section{Future Research Plans}

As a next step, we will design studies to further investigate the individual adjustment of parameters and specifically the collaborative aspects of using embodied magic lenses with the presented interaction modalities.

Moreover, we want to address the possible personal extension of magic lenses and use the benefits of graspable magic lenses as previously discussed for transparent tangibles. However, with the increase of personal mobile devices, we plan to enhance previous work (e.g., [15]) in making magic lenses mobile and using device interaction to parametrize and switch lens functions. Figure 7 shows a first setup with a lens on a mobile phone which is tracked using Optitrack IR-cameras. For this scenario, we want to combine proxemic principles with device sensor data to adjust the lens parameters and further develop our concept of personalizing magic lenses by using the properties and preferences saved on the user's personal mobile or tablet.
Additionally, further modalities should be considered for magic lenses. We could also use eye tracking to determine where the user is working and what he/she is interested to place the lens. Even more important, we plan to investigate the combination of these various interaction modalities using their individual benefits to form a coherent data exploration and manipulation setup for graphs on interactive display walls.

\section{Conclusion}

Magic lenses are an important tool in visualization. We investigate interactive graph exploration and editing and focus on magic lenses as a tool to support graph analysis on interactive display walls. A thorough analysis of the stateof-the-art resulted in open problems that we address by investigating various novel features, e.g., combination of lens functions and aspects of personalization, as well as exploring and combining diverse interaction modalities, e.g., multi-touch, tangible and body-centric interaction.

\section{Acknowledgements}

I would like to thank my supervisor Raimund Dachselt for his continuous support. Further, I thank the members of the IMLD for all their feedback and discussions during this work, especially my co-authors. The project in which this work is created is funded by the DFG (DA 1319/3-1).

\section{REFERENCES}

1. T. Ballendat, N. Marquardt, and S. Greenberg. 2010 Proxemic Interaction: Designing for a Proximity and Orientation-aware Environment. In Proc. ITS '10. ACM, 121-130. DOI : http://dx.doi.org/10.1145/1936652.1936676

2. E. A. Bier, M. C. Stone, K. Pier, W. Buxton, and T. D. DeRose. 1993. Toolglass and magic lenses: the see-through interface. In Proc. SIGGRAPH '93. ACM, 73-80. DOI : http://dx.doi.org/10.1145/166117.166126 
3. J. Brosz, M. A. Nacenta, R. Pusch, S. Carpendale, and C. Hurter. 2013. Transmogrification: Causal Manipulation of Visualizations. In Proc. UIST '13. ACM, 97-106. DOI : http://dx.doi.org/10.1145/2501988.2502046

4. W. Büschel, U. Kister, M. Frisch, and R. Dachselt. 2014. T4 - Transparent and Translucent Tangibles on Tabletops. In Proc. AVI '14. ACM, 81-88. DOI : http://dx.doi.org/10.1145/2598153.2598179

5. S. Butscher, K. Hornbæk, and H. Reiterer. 2014. SpaceFold and PhysicLenses: Simultaneous Multifocus Navigation on Touch Surfaces. In Proc. AVI '14. ACM, 209-216. DOI : http://dx.doi.org/10.1145/2598153.2598177

6. M. Frisch, U. Kister, W. Büschel, R. Langner, and R. Dachselt. 2013. Translucent Tangibles on Tabletops: Exploring the Design Space. In CHI 2013 Workshop on Blended Interaction.

7. K. Kim and N. Elmqvist. 2012. Embodied Lenses for Collaborative Visual Queries on Tabletop Displays. Information Visualization 11, 4 (Oct. 2012), 319-338. DOI : http://dx.doi.org/10.1177/1473871612441874

8. U. Kister, P. Reipschläger, and R. Dachselt. 2014. Multi-Touch Manipulation of Magic Lenses for Information Visualization. In Proc. ITS '14. ACM, 431-434. DOI : http://dx.doi.org/10.1145/2669485.2669528

9. U. Kister, P. Reipschläger, F. Matulic, and R. Dachselt. 2015. BodyLenses - Embodied Magic Lenses and Personal Territories for Wall Displays. In Proc. ITS '15. ACM, 10. DOI : http://dx.doi.org/10.1145/2817721.2817726

10. C. Lander and S. Gehring. 2014. ClothLens Demo: Simultaneous Multi-User Interaction with Shared Content on a Tabletop. In Proc. ITS '14. ACM, New York, NY, USA, 415-418. DOI : http://dx.doi.org/10.1145/2669485.2669524
11. A. Lehmann, H. Schumann, O. Staadt, and C. Tominski. 2011. Physical Navigation to Support Graph Exploration on a Large High-Resolution Display. In Advances in Visual Computing. Lecture Notes in Computer Science, Vol. 6938. Springer Berlin Heidelberg, 496-507. DOI :

http://dx.doi.org/10.1007/978-3-642-24028-7 46

12. C. Pindat, E. Pietriga, O. Chapuis, and C. Puech. 2012. JellyLens: content-aware adaptive lenses. In Proc. UIST '12. ACM, 261-270. DOI : http://dx.doi.org/10.1145/2380116.2380150

13. S. Schmidt, M. A. Nacenta, R. Dachselt, and S. Carpendale. 2010. A Set of Multi-touch Graph Interaction Techniques. In Proc. ITS '10. ACM, 113-116. DOI : http://dx.doi.org/10.1145/1936652.1936673

14. G. Shoemaker, T. Tsukitani, Y. Kitamura, and K. S. Booth. 2010. Body-centric Interaction Techniques for Very Large Wall Displays. In Proc. NordiCHI '10. ACM, 463-472. DOI : http://dx.doi.org/10.1145/1868914.1868967

15. M. Spindler, C. Tominski, H. Schumann, and R. Dachselt. 2010. Tangible Views for Information Visualization. In Proc. ITS '10. ACM, 157-166. DOI : http://dx.doi.org/10.1145/1936652.1936684

16. C. Tominski, J. Abello, F. van Ham, and H. Schumann. 2006. Fisheye Tree Views and Lenses for Graph Visualization. In Proc. IV '06. IEEE Computer Society, 17-24. DOI : http://dx.doi.org/10.1109//V.2006.54

17. C. Tominski, S. Gladisch, U. Kister, R. Dachselt, and H. Schumann. 2014. A Survey on Interactive Lenses in Visualization. In EuroVis - STARs. The Eurographics Association. DOI : http://dx.doi.org/10.2312/eurovisstar.20141172 